\title{
Male Advancement in Prenatal Hand Development
}

\author{
STANLEY M. GARN, ALPHONSE R. BURDI AND WILLIAM J. BABLER \\ Center for Human Growth and Development and Department of Anatomy, \\ The University of Michigan, Ann Arbor, Michigan 48104
}

\begin{abstract}
KEY WORDS Embryology ' Hand - Fetus - Sexual dimorphism ·
\end{abstract} Skeleton.

\begin{abstract}
Male advancement in the developing hand was evidenced by 66 grossly and microscopically normal embryos in the 15-75 $\mathrm{mm}$ crown-rump range. Male advancement was particularly pronounced in the younger (15$30 \mathrm{~mm}$ ) embryos, both in the proximal hand region including the round bones of the wrist and in the distal hand region, comprising the metacarpals and phalanges. Thirty-four additional embryos, abnormal in implantation or development or representing spontaneous abortion similarly evidenced male advancement in the early hand skeleton
\end{abstract}

In most aspects of human development, from before birth through the end of adolescence, the female is advanced over the male, by from 4 to $20 \%$ and more. This is true for ossification, as measured by the radiographic appearance of the nuclei of the round bones and epiphyses (Garn et al., '67). This is true for epiphyseal union, as seen in the hand, foot, elbow, knee, shoulder and hip (Noback, '54; Garn et al., '61). This is also true for the calcification, movement and clinical eruption of the permanent teeth, to an extent exceeding differences attributable to race (Garn et al., '58; Garn et al., '65). Female advancement in the development of the secondary sexual characteristics and in the onset of gametogenesis are well known in human biology and accepted as fact in law.

For the early prenatal period, however, during the first trimester of gestation, female advancement is not known as a fact, possibly because of the lack of comparative sex-specific embryological data. Standard works are vague on this subject, from Arey's textbook to the more specialized reports of Streeter ('49), Gray et al. ('57), and Blechschmidt ('69). Indeed, in our previously-published studies on early prenatal development of the primary (deciduous) dentition, we have found male advancement rather than the female advancement we had earlier anticipated, (Burdi et al., '70; Garn and Burdi, '71).

We therefore raise the question as to whether the deciduous teeth are unique, in suggesting male rather than female advancement in the $15-75 \mathrm{~mm}$ crownrump range, or whether male advancement is a developmental characteristic of the entire first trimester. Accordingly, therefore, we have turned our attention to the developing hand, including both the wrist area and the metacarpals and phalanges, in a prenatal developmental investigation patterned after now-familiar postnatal developmental models (cf. Garn et al., '67).

\section{METHODS AND MATERIALS}

This comparative study of early prenatal hand development is based upon two series of human embryos, all in the 15-75 mm crown-rump length range and all clearly identifiable as to sex.

The first series, upon which this paper primarily depends, comprises some $66 \mathrm{em}$ bryos, 40 of them male and 26 female, all of them from documented normal (uterine) implantations, and all apparently normal by both gross inspection and after exam ination of the histological sections. The second series, carefully separated from the first, consisted of an additional group of 34 embryos of similar crown-rump range and similarly identified as to sex, but excluded from the normal group because of abnormalities of implantation (ectopic pregnancies), or because of spontaneous abortion, or because of neural arch defects.

Sexing was accomplished by histological 
examination of gonadal development, with particular emphasis on testicular tissue. following the work of Gillman (48) and previously discussed by us (Burdi and Silvey, '69a,b).

For each specimen, both normal and abnormal, the hand area was carefully studied on both the left and the right sides, and using the total set of histological sections. The degree of histological development of each of 32 bone areas was separately rated, for each hand, and including the distal aspects of the radius and ulna, as well as the styloid process of the ulna.

For each bone area, the degree of development was rated or "staged" using the following numerical staging schedule, also pictured in figure 1 for a representative embryonic hand.

1. Mesenchymal (bone) anlage, comparable to Streeter's phase 1.

2. Chondroblast formation and appearance of cartilaginous model.

3. Chondrocyte differentiation and perichondrium formation (cf. Streeter's phase 2).

4. Chondrocyte proliferation (cf. Streetex's phase 3 ).

5. Maturation of the cartilaginous model (cf. Streeter's phase 4).

6. Chondrocyte hypertrophy (cf. Street er's phase 5).

7. Bone collar formation (beginning of true bone).

8. Vascular invasion of the bone collar.

9. Endochondral bone growth.

For the purposes of this study, individual bone ratings were summed for the carpal or wrist area, for the metacarpalphalangeal area, and totaled as follows:

A. A total score (T) for all 64 bone areas in both hands; $B$. An average score $\left(\frac{T p}{N}\right)$ for the proximal hand or wrist area, involving 13 bony areas for each hand; i.e., $N=26$; . An average score $\left(\frac{T_{d}}{N}\right)$ for the distal-hand or metacarpal-phalangeal area, or 19 tubular bones for each hand; i.e., $\mathrm{N}=38$.

This system of embryonic hand appraisal was designed to follow the postnatal developmental model, involving the tubular bones of the hand proper, the round bones of the wrist area, and the distal aspects of the bones of the forearm (Garn et al., '67). The proximal hand (wrist area) and the distal hand (metacarpals and phalanges) were separated in certain of the analyses, because the prenatal development of the round bones of the wrist and the distal aspects of the radius and ulna stops at stage 5 (maturation of the cartilaginous model).

For each embryo, then, developmental hand data consisted of the total score (T) for both hands, and the average score $\left(\frac{\mathrm{T}}{\mathrm{N}}\right)$ for the proximal and distal hand elements respectively. Dimensional information consisted of the crown-rump length (CRL). Since the raw data consisted of numerical scores, most parametric tests of significance were not appropriate, and emphasis was given to non-parametric "sign" tests. With the considerable range of crownrump lengths involved, the technique of matched-pairs was deemed especially applicable and was used in later aspects of the data analysis.

\section{FINDINGS}

In the first step of comprehensive data analysis, the basic question of sex differences in prenatal hand development was approached on a group basis, for the 40 male and 26 female embryos deemed normal by both gross and histological examination, and excluding all abnormalities of implantation and spontaneous abortions. This was accomplished initially by a simple sign test against the sex-specific trend lines relating hand scores to crown-rump length. It may be noted that 27 of the 40 male embryos $(67 \%)$ were advanced over size-comparable female embryos, while only 9 of the 26 females ( $35 \%$ ) were advanced over size-comparable males. Compared with each other, and with the chance (50:50) distribution, these sex differences were significant by the stochastic chisquared test, with values of $\chi^{2}$ as high as 19.0 .

Quantification was then applied, separately for embryos in the 15-30 $\mathrm{mm}$ crown-rump range and for those in the larger (30-75 $\mathrm{mm}$ ) crown-rump range. To do this both the total hand score $(T)$, the sum of individual bone "stages," and the average hand score $\left(\frac{T}{N}\right)$ for the proximal and distal hand regions were expressed 


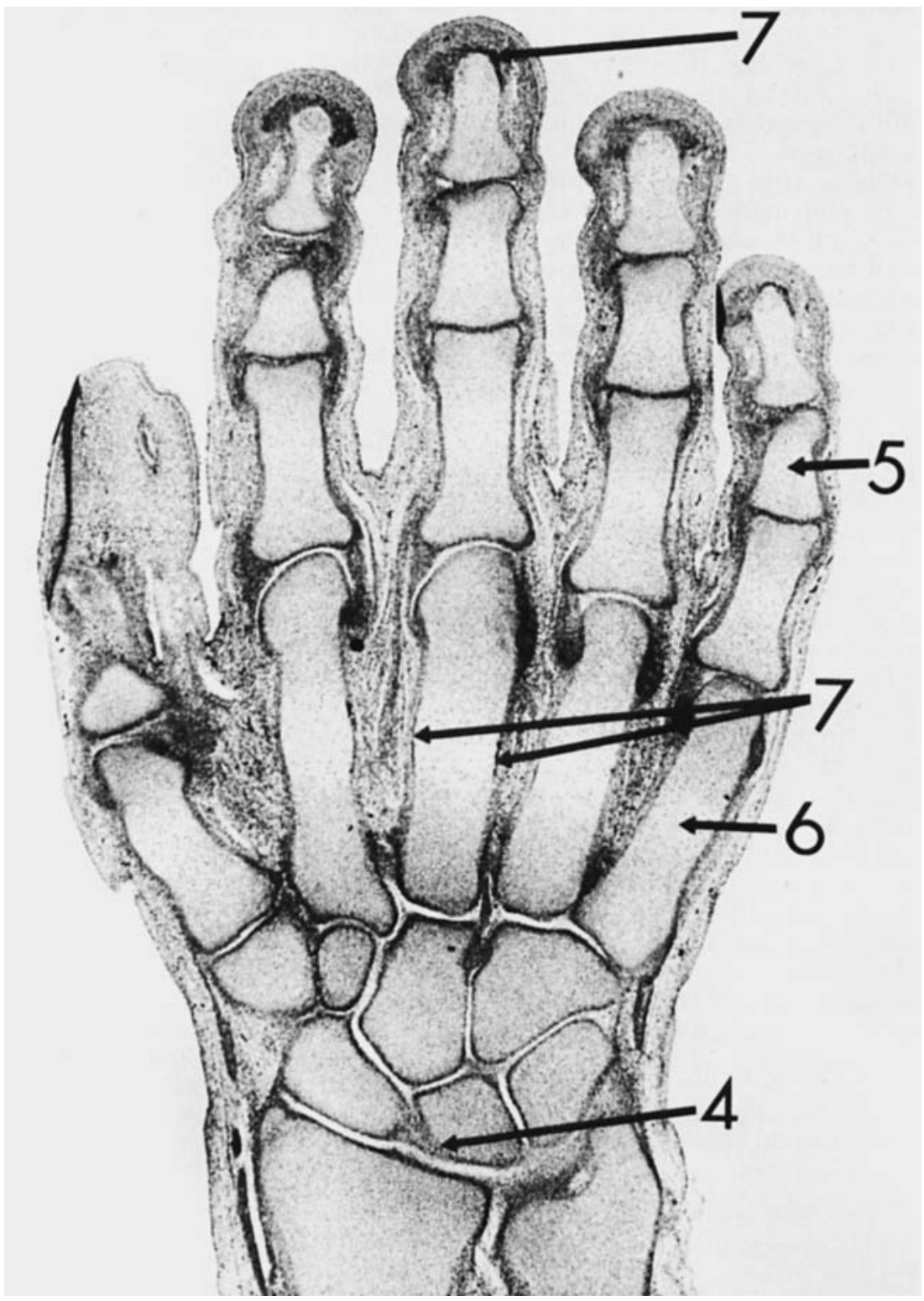

Fig. 1 Photomicrograph of the right hand of a $42 \mathrm{~mm}$ CRL male embryo showing 4 of the 9 developmental stages described in the text. Indicated by arrows are: stage 4, chondrocyte proliferation and still-incomplete perichondrial margin (as in the lunate and the styloid process of the ulna); stage 5, (cartilaginous maturation including well-formed perichondrium as in the remaining carpals and middles); stage 6, chondrocyte hypertrophy (as in the light area on metacarpal 5) and stage 7 , bone formation (as in the bone collar on the 2 nd, 3rd, and 4 th metacarpals and bone formation on the tufts of the distals).

For the hand shown here the total score $(T)$, that is the sum of stages attained by individual skeletal elements is 174 . In contrast, the average score $\left(\frac{T}{N}\right)$ for the proximal hand (comprising the distal radius and ulna and carpals) is 4.7 , and the average score for the distal hand (metacarpals and phalanges) is 6.0. Note that distal I is out of the plane of the section. 
relative to crown-rump length, on an individual basis. What was measured was embryonic development for the entire hand skeleton, and for the two hand regions, uniformly expressed per millimeter of crown-rump length.

This analysis, then performed on a grouped basis, and using familiar $t$ statistics, confirmed male advancement in the 15-30 mm CRL range, as shown in table 1 , and to a lesser extent in the $30-75 \mathrm{~mm}$ CRL. The early $15-30 \mathrm{~mm}$ sex differences in absolute and relative hand development were highly significant, as shown. The latter $30-75 \mathrm{~mm}$ sex differences were not statistically significant, though in the same direction (i.e., male advancement) as shown in the table. For all 66 embryos deemed normal by both gross and histological examination, hand development per $\mathrm{mm}$ of CRL was considerably advanced in the males. However, the bulk of the male advancement was concentrated in the earlier $15-30 \mathrm{~mm}$ crown-rump range, where hand development was approximately $50 \%$ greater per millimeter of crown-rump length.

In the third stage of data analysis, malefemale differences in early prenatal hand development were analyzed on an individual basis, using the matched-pair technique to correct for differences in crownrump length (CRL). To do this, male and female embryos were length-matched to within $\pm 6 \mathrm{~mm}$, and differences in total hand score (T) and the proximal and distal average scores $\left(\frac{T}{N}\right)$ calculated and recorded as in table 2 . The question was the extent of sex difference in skeletal (hand) development remaining after close matching for crown-rump length.

As shown in table 2, the pairs were matched to an average length difference of zero, but despite the close match in length, the males were still systematically advanced in (a) total hand score, (b) average score for the proximal hand area and (c) average hand score for the distal hand area. The sex differences in development remaining after length-matching were reasonably large, approximately $10 \%$, the difference taking $D$ as a percent of the mean total score or mean average score. The sex differences remaining after lengthmatching were all highly significant, by $t$ test, using the standard error of the difference between matched pairs. Moreover, the sex differences in hand development were nearly as large when the pairs of embryos were rematched to within \pm 4 $\mathrm{mm}$, as also shown in table 2.

In the last step of the data analysis, attention was directed to the 34 additional embryos deemed abnormal because they were spontaneously aborted, abnormally implanted or defective by gross or microscopic examination. These abnormal embryos were divided into three groups despite the small samples that then ensued. Nevertheless, after division by sex and by group (spontaneously aborted, ectopic pregnancies and neural arch defects), male advancement was still evident. On the group basis, male embryos with defects of implantation or spontaneously aborted embryos showed systematically greater total hand scores and distal hand scores than length-matched female embryos of the same crown-rump length groupings.

TABLE 1

Comparative hand development of smaller (15-30 $\mathrm{mm}$ ) and larger (31-75 $\mathrm{mm}$ ) first trimester fetuses

\begin{tabular}{|c|c|c|c|c|c|}
\hline \multirow{2}{*}{$\begin{array}{l}\text { Crown-rump } \\
\text { length ( } \mathrm{mm})\end{array}$} & \multirow[b]{2}{*}{ Sex } & \multirow[b]{2}{*}{$\mathbf{N}$} & \multicolumn{3}{|c|}{ Hand stage pex $\mathrm{mm} \mathrm{CRL}$} \\
\hline & & & $\begin{array}{l}\text { Total } \\
\text { hand } 1\end{array}$ & $\begin{array}{c}\text { Proximal } \\
\text { hand } 2\end{array}$ & $\begin{array}{l}\text { Distal } \\
\text { hand }{ }^{2}\end{array}$ \\
\hline $15-30$ & $\mathbf{M}$ & 16 & 9.13 & 0.13 & 0.14 \\
\hline $15-30$ & $\mathbf{F}$ & 14 & 6.37 & 0.09 & 0.09 \\
\hline$t$ & & & $3.36^{3}$ & 4.203 & 3.33 \\
\hline $31-75$ & $\mathbf{M}$ & 24 & 8.19 & 0.10 & 0.13 \\
\hline $31-75$ & $\mathrm{~F}$ & 12 & 7.59 & 0.09 & 0.13 \\
\hline$t$ & & & 1.05 & 1.10 & 0 \\
\hline
\end{tabular}

1 Total hand score divided by crown-rump length

2 Average stage per skeletal unit divided by crown-rump length.

${ }^{3}$ Significant at $\boldsymbol{p}=0.05$ or better. 
TABLE 2

Male hand advancoment in fetuses matched for coown-rump length

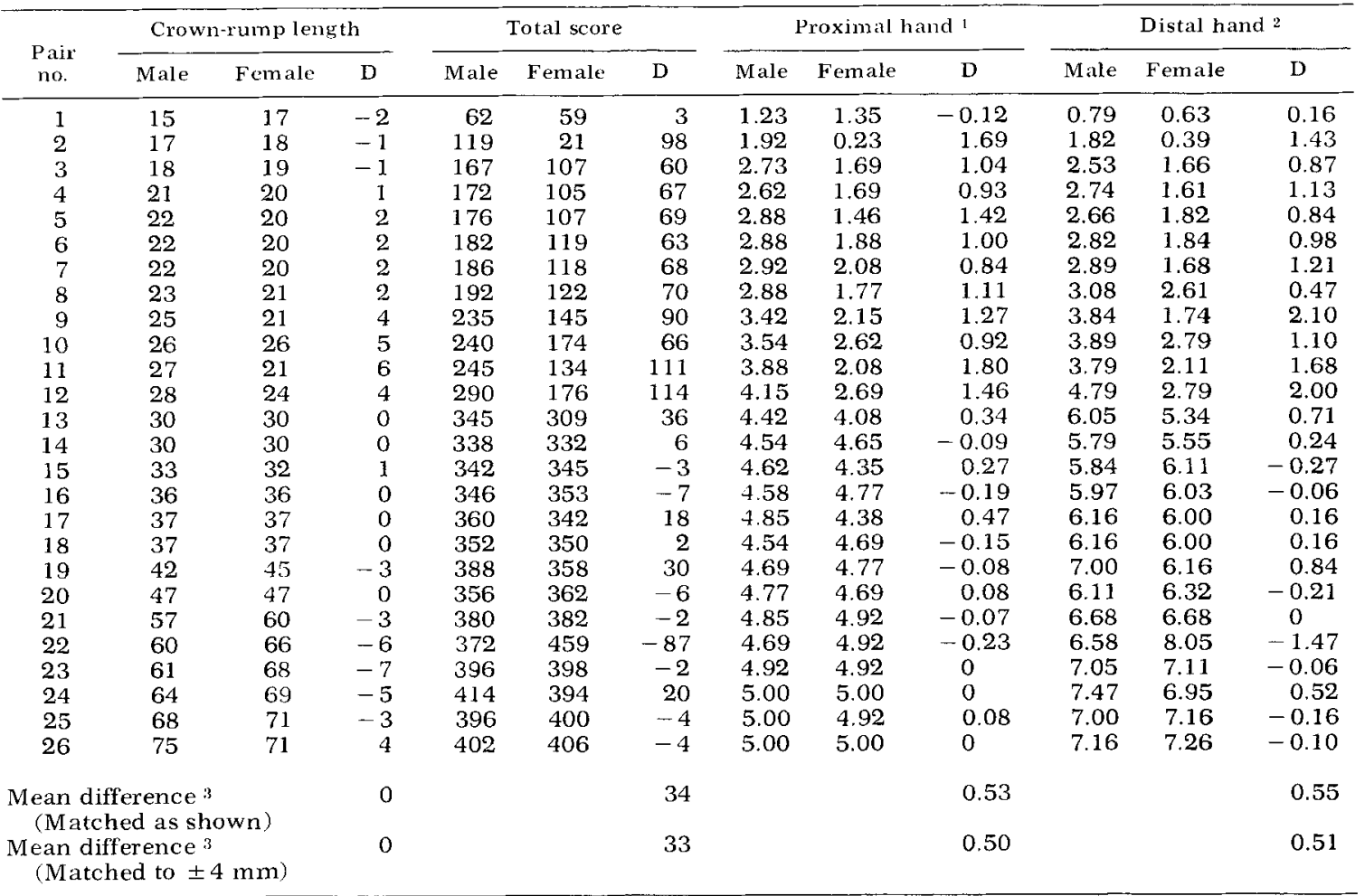

1 Average for 13 proximal elements.

2 Average value for 19 distal elements.

is All mean male-female differences (D) in hand development significant at $p=0.001$.

This was also true for developmentally abnormal embryos in the smaller (15-30 $\mathrm{mm}$ ) crown-rump range. Thus, embryos derived from tubal implantations, embryos spontaneously aborted and developmentally-abnormal embryos together showed male advancement in the $15-30 \mathrm{~mm}$ crown-rump range.

Matched-pair comparisons of abnormal embryos and those normal by gross and histological examination but abnormal in implantation also evidenced male advancement. For six pairs of developmentally abnormal embryos, size-matched within $\pm 1 \mathrm{~mm}$, the male embryos were generally advanced in hand development though not significantly so $(t=1.21)$. For six pairs of normal-appearing embryos derived from ectopic pregnancies or spontaneous abortions, male advancement was systematic, and though matched very closely to within $\pm 2 \mathrm{~mm}$ on the average, there was a sex difference of some 49 units in total hand score, $(t=3.83)$ highly significant by any reasonable measure. For all 12 pairs of size-matched embryos in the 15$30 \mathrm{~mm}$ size range, deemed abnormal by virtue of implantation or examination, the sex difference was marked and the male advancement statistically significant.

Therefore, whether comparing hand development of male and female embryos of the same size, or expressing prenatal hand development per unit of crown-rump length, for the total hand or for the proximal and distal hand elements, or using the matched-pair technique, and for both normal and abnormal embryos as devised above, male advancement in the developing hand was seen to be a consistent feature, primarily in the $15-30 \mathrm{~mm}$ crownrump range. 


\section{DISCUSSION}

The findings in this new study are both clear-cut and simple to describe. They show that, in the 15-75 $\mathrm{mm}$ crown-rump range, the male embryo is further ahead (i.e., advanced) in the development of the hand skeleton, and up to the time of appearance of calcified bone tissue. This is true separately for the proximal hand alone (including the carpals), for the distal hand alone (the metacarpals and phalanges) and, of course, it is then true for the hand-wrist area as a whole. If we express early hand development as a total score, the sum of the stages achieved by individual bone areas, and in relation to crown-rump length, then we find that the male embryo is approximately $10 \%$ advanced over the female embryo of equal length.

Conversely, for constant stage of hand development (measured as a total score), the male embryo is then shorter in crownrump length, by some $10 \%$ in that stem dimension. This generalization holds true when the proximal hand-wrist area is considered alone, with its 13 skeletal elements, and when the distal hand area is considered alone, with its 19 tubular bones. Either the 15-75 mm male embryo is advanced in the development of the hand skeleton, for given crown-rump length, or the female embryo is dimensionally long for her developmental status during the first trimester. Either way, there is a rather large sex difference in early development and developmental timing of the hand skeleton, more so in the smaller 15-30 mm crown-rump range than in the larger and later $30-75 \mathrm{~mm}$ crownrump range.

This finding of male advancement for constant length does not have postnatal parallels in (1) ossification timing, (2) epiphyseal union, (3) development of the permanent dentition nor (4) in sexual maturation. It is, however, consistent with our previously-published data on palatal closure (Burdi and Silvey, '69a,b), and our findings on early prenatal dental development (Garn and Burdi, '71). It is, moreover, consistent with Infante's recent findings on early postnatal deciduous tooth emergence (Infante, '73). During the first trimester it is the male and not the female that is advanced in both skeletal (hand) and dental development, as our studies on 20 deciduous teeth and 32 elements of the hand skeleton together show.

The implications of these continuing findings are numerous. To the extent that there are still stage-specific developmental insults, one might expect critical timing to be markedly different for male and female embryos. To the extent that such insults are time-specific, one might expect the sexes to differ considerably in the stage of embryological development attained.

The data here set forth in tabular form, showing developmental differences in sizematched embryos and in the data analyses employed point out several important new generalizations about early human prenatal development. First, they document the excess of male embryos, in spontaneous abortions. Second, they show that male embryos are developmentally different from female embryos of comparable crownrump lengths, both in apparently normal embryos and in those of abnormal implantation as well as those abnormal by gross and histologic examination. Third, they show the extent of early prenatal developmental variability at constant length, akin to the postnatal situation with which we are all familiar, and hinting at prenatal origins for later size differences. Finally, they show that the proximal hand-wrist area, including the carpus, and the distal hand area, comprising the metacarpals and phalanges evidence comparable male advancement for constant crown-rump length. In this way the way is open for prenatal hand standards, necessarily sexspecific, such as have long been employed in postnatal developmental assessment.

\section{ACKNOWLEDGMENTS}

This investigation was supported, in part, by NIH research grant number DE 03443 from the National Institute of Dental Research and by the Beatrice Stanton Medical Research Fund, the University of Michigan Medical School. The manuscript was completed by Miss Dixie L. Farquharson.

\section{LITERATURE CITED}

Blechschmidt, E. 1969 The early stages of human limb development. In: Limb Development 
and Deformity: Problems of Evaluation and Rehabilitation. C. A. Swinyard, ed., C. C Thomas, Springfield, pp. 24-56.

Burdi, A. R., S. M. Garn and R. L. Miller 1970 Developmental advancement of the male dentition in the first trimester. J. Dent. Res., 49: 889.

Burdi, A. R., and R. G. Silvey 1969a Sexual differences in closure of the human palatal shelves. Cleft Pal. J., 6:1-7.

$1969 \mathrm{~b}$ The relation of sex-associated facial profile reversal and stages of human palatal closure. Teratology, 2 : 297-304.

Garn, S. M., and A. R. Burdi 1971 Prenatal ordering and postnatal sequence in dental de velopment. J. Dent. Res., 50: 1407-1414.

Garn, S. M., A. B. Lewis and R. M. Blizzard 1965 Endocrine factors in dental development. J. Dent. Res., 44: 243-258.

Garn, S. M., A. B. Lewis, K. Koski and D. L. Polacheck 1958 The sex differences in tooth calcification. J. Dent. Res, , 37:561-567.

Garn, S. M., C. G. Rohmann and B. Apfelbaum 1961 Complete epiphyseal union of the hand. Am. J. Phys. An throp., 19: 365-372.

Garn, S. M., C. G. Rohmann and F. N. Silverman
1967 Radiographic standards for postnatal ossification and tooth calcification. Med. Radiog. and Photog., 43: 45-66.

Gillman, J. 1948 The development of the gonads in man, with a consideration of the role of fetal endocrines and histogenesis of ovarian tumors. Carnegie Contributions to Embryology $32: 81-131$.

Gray, D. J., E. Gardner and R. O'Rahilly 1957 The prenatal development of the skeleton and joints of the human hand. Am. J. Anat., 101 169-224.

Infante, P. F. 1973 Epidemiologic studies of the relation between deciduous tooth eruption and child growth. Thesis presented to the School of Public Health, The University of Michigan. Ann Arbor, Michigan

Noback, C. R. 1954 The appearance of ossifi cation centers and the fusion of bones. Am. J Phys. Anthrop., 12:63-69.

Streeter, G. L. 1949 Developmental horizons in human embryos (fourth issue). A review of the histogenesis of cartilage and bone. Carnegie Inst. Wash. Pub. 583, Contrib. to Embryol. 33: 149-168. 